\title{
SUPPORTS OF CONTINUOUS FUNCTIONS
}

\author{
BY \\ MARK MANDELKER( $\left.{ }^{1}\right)$
}

\begin{abstract}
Gillman and Jerison have shown that when $X$ is a realcompact space, every function in $C(X)$ that belongs to all the free maximal ideals has compact support. A space with the latter property will be called $\mu$-compact. In this paper we give several characterizations of $\mu$-compact spaces and also introduce and study a related class of spaces, the $\psi$-compact spaces; these are spaces $X$ with the property that every function in $C(X)$ with pseudocompact support has compact support. It is shown that every realcompact space is $\psi$-compact and every $\psi$-compact space is $\mu$-compact. A family $\mathscr{F}$ of subsets of a space $X$ is said to be stable if every function in $C(X)$ is bounded on some member of $\mathscr{F}$. We show that a completely regular Hausdorff space is realcompact if and only if every stable family of closed subsets with the finite intersection property has nonempty intersection. We adopt this condition as the definition of realcompactness for arbitrary (not necessarily completely regular Hausdorff) spaces, determine some of the properties of these realcompact spaces, and construct a realcompactification of an arbitrary space.
\end{abstract}

1. Introduction. The support of a real continuous function $f$ on a topological space $X$ is the closure of the set of points in $X$ at which $f$ does not vanish. Gillman and Jerison have shown that when $X$ is a realcompact space, the functions in $C(X)$ with compact support are precisely the functions which belong to every free maximal ideal in $C(X)$. This result, and other general background material, may be found in our basic reference [GJ].

A space with the property of the Gillman-Jerison result will be said to be $\mu$ compact. Other writers have shown that discrete spaces (Kaplansky [ $\mathrm{K}_{1}$, Theorem 28]), $P$-spaces (Kohls [ $\mathrm{K}_{2}$, Theorem 3.9]), and spaces that admit complete uniform structures (Robinson $\left[\mathbf{R}_{1}\right]$ ) are $\mu$-compact. Examples given in [GJ] show that not every space is $\mu$-compact, and not every $\mu$-compact space is realcompact.

In this paper we show that a third class of spaces may be interpolated between the realcompact and $\mu$-compact. This class, of $\psi$-compact spaces, consists of those spaces $X$ for which every function in $C(X)$ with pseudocompact support has compact support. Examples will be given to show that the three classes of spaces are distinct. Every $P$-space (hence every discrete space) and every space that admits a

Received by the editors December 19, 1969.

AMS 1970 subject classifications. Primary 46E25, 54C40, 54D60; Secondary 54D10, 54D30.

Key words and phrases. Support, continuous function, compact support, pseudocompact support, free maximal ideal, realcompact space, $\mu$-compact space, $\psi$-compact space, relatively pseudocompact subset, stable family, round subset, realcompactification.

(1) The author gratefully acknowledges support from the National Science Foundation under Grant NSF-GP-9630. 
complete uniform structure is $\psi$-compact; thus the result that every $\psi$-compact space is $\mu$-compact is a common extension of the results of Kaplansky, Kohls, and Robinson. We also give several characterizations of $\mu$-compact spaces.

A characterization of realcompact spaces is obtained here which permits the concept of realcompactness to be extended to spaces which are not necessarily completely regular Hausdorff, and for which the main results of this paper and many of the properties of realcompact spaces are still valid. Defining a family $\mathscr{F}$ of subsets of a space $X$ to be stable if every function in $C(X)$ is bounded on some member of $\mathscr{F}$, we show that a space $X$ is realcompact if and only if every stable family of closed subsets with the finite intersection property has nonempty intersection. For completely regular Hausdorff spaces we show the equivalence of this condition and the usual definition of realcompactness, while for arbitrary spaces we adopt this condition as the definition of realcompactness. We determine some of the properties of these realcompact spaces and construct a realcompactification of an arbitrary space.

The author wishes to thank L. Gillman, D. G. Johnson, D. Plank, and the referee for helpful comments concerning this paper.

2. Supports. In any topological space $X$, the support of a function $f$ in $C(X)$ is the set $S(f)=\operatorname{cl}_{X}\{x \in X: f(x) \neq 0\}$. Any subset of $X$ that is the support of some function in $C(X)$ is called a support in $X$. An arbitrary closed set in $X$, even a zeroset, need not be a support. For example, a nonempty nowhere dense set is never a support.

We are interested in functions with compact support. A weaker condition on a subset $S$ of $X$ is that it be pseudocompact, i.e., every function in $C(S)$ is bounded. Still weaker is the following condition.

Definition. A subset $S$ of a space $X$ is relatively pseudocompact in $X$ if every function in $C(X)$ is bounded on $S$.

Theorem 2.1 below shows that for supports this condition is equivalent to pseudocompactness. The equivalence does not hold for arbitrary subsets of $X$, not even for closed subsets. For example, the Tychonoff plank $T$ (see [GJ, 8.20]) is a pseudocompact space, and hence every subset is relatively pseudocompact; however, the right edge $N$ (which is closed in $\mathbf{T}$ ) is a countably infinite discrete space, and thus not pseudocompact. In a normal space, of course, the two conditions are equivalent for any closed subset.

The following theorem says that if a support $S$ in $X$ admits an unbounded continuous function, then some unbounded continuous function on $S$ may be extended continuously over $X$.

THEOREM 2.1. In any topological space $X$, any relatively pseudocompact support is pseudocompact.

Proof. Let $S$ be a support in $X$ that is not pseudocompact; thus $S=\operatorname{cl}_{X}(X-Z)$ for some zero-set $Z$ in $X$, and there is a function $h \geqq 1$ in $C(S)$ that is unbounded, 
hence unbounded on $X-Z$. It follows from [GJ, 1.20] that $X-Z$ contains a $C$-embedded subset $D$ of $S$ on which $h$ is unbounded, and thus from [GJ, 1.18] that $D$ is completely separated from the zero-set $W=Z \cap S$ in $S$; hence we may choose $g \in C(S)$ with $g=0$ on $D$ and $g=1$ on $W$. Put $f=(1 / h) \vee g$ on $S$ and $f=1$ on $X-S$. Clearly $f \in C(X), f>0$, and $1 / f$ is unbounded on $D$. Thus $S$ is not relatively pseudocompact.

COROLlary 1. Any support in a pseudocompact space is also pseudocompact.

COROllary 2. For any topological space $X$, the family of all real-valued continuous functions with pseudocompact support is an ideal (possibly improper) in $C(X)$; it is all of $C(X)$ if and only if $X$ is pseudocompact.

When $X$ is a completely regular Hausdorff space, it is clear that a subset $S$ of $X$ is relatively pseudocompact if and only if $\operatorname{cl}_{v X} S$ is compact, where $v X$ is the Hewitt realcompactification of $X$ (see [GJ, 8E]). Thus, in the case of a completely regular Hausdorff space, Theorem 2.1 follows from a result of A. W. Hager and D. G. Johnson $\left[\mathrm{H}_{1}\right.$, p. 96], $\left[\mathrm{C}_{1}\right.$, Theorem 4.1], which shows, in fact, that in this case, if the closure of any open set is relatively pseudocompact, then it is pseudocompact. In the case of a support, the proof given in $\left[\mathrm{C}_{1}\right]$ applies to an arbitrary topological space; the proof given above, however, seems simpler. Also, the result as stated above for supports is sufficient for use in the proof of Theorem 4.4 in $\left[C_{1}\right]$. Other properties of relatively pseudocompact sets may be found in $\left[\mathrm{N}_{2}\right]$.

THEOREM 2.2. Let $X$ be any topological space. Every function in $C(X)$ that belongs to all the free maximal ideals has pseudocompact support.

Proof. Let $f \in C(X)$ with nonpseudocompact support $S$. Thus $S$ is not relatively pseudocompact and $X-Z(f)$ contains a $C$-embedded subset $D$ of $X$ on which some $g \in C(X)$ is unbounded. Since $D$ is completely separated from $Z(f)$, there is a zero-set $W$ in $X$ with $D \subseteq W$ and $W \cap Z(f)=\varnothing$. For every $n \in N$, the zero-set $Z_{n}$, of points in $X$ at which $|g| \geqq n$, meets $W$, and thus there is a $z$-ultrafilter $\mathscr{M}$ on $X$ containing $W$ and all the sets $Z_{n}$. Obviously $\bigcap_{n} Z_{n}=\varnothing$; hence $\mathscr{M}$ is free and $f$ does not belong to the corresponding free maximal ideal.

In the case of a completely regular Hausdorff space, this result is essentially the content of the lemma in $\left[R_{2}\right]$. In fact, in this case the proof may be simplified by noting that $W$ is not compact, and hence belongs to some free $z$-ultrafilter, by the following result of [GJ, 4.10].

(a) A zero-set in a completely regular Hausdorff space is compact if and only if it belongs to no free $z$-filter.

The proof above actually provides the following analogue for arbitrary spaces of the sufficiency in (a). A zero-set that belongs to no free $z$-filter is relatively pseudocompact. The converse of this result, however, is not true; for example, in any pseudocompact, noncompact, completely regular Hausdorff space $X$, the set $X$ itself is a relatively pseudocompact zero-set that does belong to a free $z$-filter. 
3. $\psi$-compact spaces. It is easily seen (see, e.g., [GJ, 7E]) that every function in $C(X)$ with compact support belongs to every free maximal ideal in $C(X)$. When the converse holds we shall call $X \mu$-compact. Thus

Definition. A space $X$ is $\mu$-compact if any function in $C(X)$ that belongs to all the free $\mu$ aximal ideals has compact support.

In [GJ, 8.19] it is shown that every realcompact space is $\mu$-compact, and a counterexample to the converse is given. We show below that between these classes of spaces we may interpolate the following third class.

Definition. A space $X$ is $\psi$-compact if every function in $C(X)$ with $\psi$ eudocompact support has compact support.

THEOREM 3.1. For any space $X$, each of the following conditions implies the next. The converses are not true.

(1) $X$ is realcompact.

(2) $X$ is $\psi$-compact.

(3) $X$ is $\mu$-compact.

Proof. Every closed subspace of a realcompact space is realcompact [GJ, 8.10], and any pseudocompact realcompact space is compact [GJ, 5H]. Hence (1) implies (2). Theorem 2.2 shows that (2) implies (3). The counterexamples to the converses are given below.

A $P$-space is a completely regular Hausdorff space in which every $G_{\delta}$ is open. Every discrete space is a $P$-space, but not conversely; see [GJ, $4 \mathrm{JKN}$ ].

THEOREM 3.2. Every P-space and every space that admits a complete uniform structure is $\psi$-compact.

Proof. Every subspace of a $P$-space is a $P$-space, and every pseudocompact $P$-space is finite [GJ, $4 \mathrm{~K}$ ].

Every closed subspace of a complete space is complete, and every pseudocompact complete space is compact [GJ, 15CQ].

If $X$ is metrizable, then since it admits a complete structure [GJ, 15.24], it is $\psi$-compact. However, this also follows directly from the fact that any pseudocompact metrizable space is compact.

Since $X$ itself is always a support, the following is immediate.

THEOREM 3.3. Every pseudocompact $\psi$-compact space is compact.

EXAMPLe 1. The $P$-space $X$ constructed in [GJ, 9L] is not realcompact; hence not every $\psi$-compact space is realcompact. Since $X$ is of nonmeasurable cardinal, Shirota's Theorem [GJ, 15.20] shows that $X$ does not admit a complete uniform structure. Thus not every $\psi$-compact space admits a complete uniform structure. Hence, if measurable cardinals exist, the class of spaces that admit complete structures lies properly between the classes of realcompact spaces and $\psi$-compact spaces. 
EXAMPLE 2. The space of countable ordinals is $\mu$-compact, as noted in [GJ, 8.19], but since it is pseudocompact (and not compact), it is not $\psi$-compact; hence not every $\mu$-compact space is $\psi$-compact. This also shows that a pseudocompact $\mu$ compact space need not be compact (cf. Theorem 3.3).

4. $\mu$-compact spaces. In this section we give a few characterizations of $\mu$ compact spaces. One of the characterizations involves the following concept. When $X$ is a completely regular Hausdorff space, a subset $A$ of the Stone-Čech compactification $\beta X$ is said to be round if whenever $\mathrm{cl}_{\beta X} Z$ contains $A$, where $Z \in Z(X)$, then $\mathrm{cl}_{\beta X} Z$ is a neighborhood of $A$ (see [M]).

THEOREM 4.1. $A$ subset $A$ of $\beta X$ is round if and only if, for any $Z \in Z(X)$,

$$
\operatorname{int}_{A}\left(\mathrm{cl}_{\beta X} Z \cap A\right)=\text { int }_{\beta X} \operatorname{cl}_{\beta X} Z \cap A \text {. }
$$

Proof. Necessity. Let $p \in \operatorname{int}_{A}\left(\mathrm{cl}_{\beta X} Z \cap A\right)$. Choose $W \in Z(X)$ such that $p \in A-\operatorname{cl}_{\beta X} W \subseteq \operatorname{cl}_{\beta X} Z \cap A$. Thus $A \subseteq \operatorname{cl}_{\beta X} Z \cup \mathrm{cl}_{\beta X} W=\mathrm{cl}_{\beta X}(Z \cup W)$, and by hypothesis we have $A \subseteq \operatorname{int}_{\beta X} \operatorname{cl}_{\beta X}(Z \cup W)$. Hence

$$
p \in \operatorname{int}_{\beta X} \operatorname{cl}_{\beta X}(Z \cup W) \cap\left(\beta X-\mathrm{cl}_{\beta X} W\right) \subseteq \mathrm{cl}_{\beta X} Z,
$$

so $p \in$ int $_{\beta X} \mathrm{cl}_{\beta X} Z$. Thus int ${ }_{A}\left(\mathrm{cl}_{\beta X} Z \cap A\right) \subseteq$ int $_{\beta X} \mathrm{cl}_{\beta X} Z \cap A$. The opposite inclusion is immediate.

Sufficiency. Let $A \subseteq \mathrm{cl}_{\beta X} Z$ for some $Z \in Z(X)$. Then $A=\operatorname{int}_{A}\left(\mathrm{cl}_{\beta X} Z \cap A\right)$ $=$ int $_{\beta X} \mathrm{cl}_{\beta X} Z \cap A$ and hence $\mathrm{cl}_{\beta X} Z$ is a neighborhood of $A$.

THEOREM 4.2. For any completely regular Hausdorff space $X$, the following are equivalent.

(1) $X$ is $\mu$-compact.

(2) $\beta X-X$ is a round subset of $\beta X$.

(3) For any $Z \in Z(X)$, int ${ }_{\beta X-X}\left(\mathrm{cl}_{\beta X} Z-X\right)=\operatorname{int}_{\beta X} \mathrm{cl}_{\beta X} Z-X$.

(4) Every cozero-set with noncompact closure contains a noncompact zero-set.

(5) Any function in $C(X)$ that belongs to all the free maximal ideals has realcompact support.

Proof. The equivalence of (1) and (2) was noted in [M, Theorem 5.1]. The equivalence of (2) and (3) is a special case of Theorem 4.1. A function belongs to every free maximal ideal if and only if its zero-set meets every noncompact zero-set [GJ, 4E.2]; hence (1) and (4) are equivalent. The equivalence of (1) and (5) follows from Theorem 2.2 .

Condition (3) cannot be extended by replacing the sets $\operatorname{cl}_{\beta X} Z$, for $Z \in Z(X)$ (which form a base for the closed sets in $\beta X$ ) by arbitrary closed sets in $\beta X$. For example, the condition does not hold for the closed subset $\beta \mathbf{R}-\mathbf{R}$ of $\beta \mathbf{R}$.

The fact that (1) implies (3), and the method used in the proof of Theorem 4.1, is due to Donald Plank [P, 5.1]. 
Other characterizations of $\mu$-compact spaces may be found in $\left[R_{2}\right]$. The condition (5) above is essentially condition (2) of $\left[R_{2}\right]$.

It was noted in $\S 3$ that a metrizable space is $\psi$-compact (and hence $\mu$-compact). That it is $\mu$-compact also follows directly from condition (4) of Theorem 4.2 : if $U$ is open and $\operatorname{cl}_{X} U$ is noncompact, then $\operatorname{cl}_{X} U$ contains a sequence $\left\{x_{n}\right\}_{n}$ with no convergent subsequence; thus a set $\left\{y_{n}: n \geqq 1\right\}$ of points of $U$ chosen so that $\rho\left(y_{n}, x_{n}\right) \leqq 1 / n$ is a noncompact closed set in $X$.

In Example 2 we used the fact, noted in [GJ, 8.19], that the space $\mathbf{W}$ of countable ordinals is $\mu$-compact. This is also easily seen by using condition (2) of Theorem 4.2. If $f \in C(\mathbf{W})$ and $\omega_{1} \in \mathrm{cl}_{\beta} \mathbf{W} Z(f)$, then $f$ must vanish on a tail and hence $\mathrm{cl}_{\beta} \mathbf{W} Z(f)$ is a neighborhood of $\omega_{1}$. Thus $\left\{\omega_{1}\right\}=\beta \mathbf{W}-\mathbf{W}$ is a round subset of $\beta \mathbf{W}$.

We saw in Example 2 that pseudocompactness and $\mu$-compactnesss together do not imply compactness. However, in the following special case, the implication does hold.

THEOREM 4.3. Let $Y$ be any locally compact, $\sigma$-compact metrizable space (e.g. the line), and let $Y \subset X \subseteq \beta Y$. If $X$ is pseudocompact and $\mu$-compact, then $X=\beta Y$.

Proof. Let $X$ be $\mu$-compact and $X \neq \beta Y$. Since $Y$ is a cozero-set in $\beta Y$, it is also a cozero-set in $X$. Choose a noncompact zero-set $Z$ in $X$ with $Z \subseteq Y$. Since $Y$ is metrizable, $Z$ is $C^{*}$-embedded in $Y$ and is not pseudocompact. Hence $Z$ is $C^{*}$ embedded in $X$ and since it is a zero-set in $X$, it is $C$-embedded in $X$. Hence $X$ is not pseudocompact.

5. Realcompact spaces. Heretofore, these spaces have been defined only in the context of completely regular Hausdorff spaces-see [GJ, 5.9 and 8.4].

By means of the characterization of realcompact spaces given in Theorem 5.1 below, we may (consistently) define realcompactness for arbitrary topological spaces. Under the definition given, the properties of realcompact spaces needed for the proof of Theorem 3.1 remain valid, and hence Theorem 3.1 is true in the general case. The definitions of $\psi$-compact and $\mu$-compact spaces apply to the general case as given, as does characterization (5) of $\mu$-compact spaces given in Theorem 4.2.

Definition. We shall say that a family $\mathscr{F}$ of subsets of a space $X$ is stable if every function $f$ in $C(X)$ is bounded on some member of $\mathscr{F}$.

THEOREM 5.1. A completely regular Hausdorff space is realcompact if and only if every stable family of closed subsets with the finite intersection property has nonempty intersection.

Proof. Let $X$ be realcompact and suppose $\mathscr{F}$ is a family of closed subsets of $X$ with the finite intersection property, but with empty intersection. Then the family $\left\{\mathrm{cl}_{\beta X} F: F \in \mathscr{F}\right\}$ of closed subsets of $\beta X$ has the finite intersection property, and we may choose $p \in \beta X$ with $p \in \mathrm{cl}_{\beta X} F$ for all $F \in \mathscr{F}$. It follows that $p \in \beta X-X$ and thus there is $f \in C(X)$ with $f^{*}(p)=\infty$. Thus $f$ is unbounded on each member of $\mathscr{F}$, and hence $\mathscr{F}$ is not stable. 
Now assume that $X$ is not realcompact. Thus there is $p \in \beta X-X$ such that $f^{*}(p)$ is finite for all $f \in C(X)$. Let $\mathscr{M}$ be the (free) $z$-ultrafilter on $X$ that converges to $p$; it consists of all zero-sets $Z$ in $X$ such that $p \in \operatorname{cl}_{\beta X} Z$. Thus $\mathscr{M}$ is a family of closed subsets of $X$ with the finite intersection property and empty intersection. Let $f \in C(X)$. Since $f^{*}(p)$ is finite, $f^{*}$ is bounded on some zero-set neighborhood $W$ of $p$ in $\beta X$. Since $p \in \operatorname{cl}_{\beta X}(W \cap X)$, we have $W \cap X \in \mathscr{M}$ with $f$ bounded on $W \cap X$. It follows that $\mathscr{M}$ is stable.

This result may perhaps be considered a partial reply to Hewitt's comment: “These spaces, which we have called $Q$-spaces, are characterized by no topological property so simple as bicompactness; indeed, their description may be considered somewhat recondite," $\left[\mathrm{H}_{5}\right.$, p. 85]. There are of course other characterizations of a realcompact space, but this characterization, when used as a definition in the general case, allows us to extend Theorem 3.1. Specifically, the condition of this characterization is hereditary for closed subspaces, and, in the presence of pseudocompactness, implies compactness. Other characterizations of realcompact spaces are found in $[\mathrm{GJ}],\left[\mathrm{H}_{3}\right],\left[\mathrm{H}_{5}\right],\left[\mathrm{N}_{1}\right]$, and $[\mathrm{W}]$. An internal characterization has recently been found by Douglas Harris $\left[\mathrm{H}_{2}\right]$. The author wishes to thank Professor M. C. Rayburn for pointing out that a special form of Theorem 5.1 was observed in $[\mathrm{F}]$.

The characterization given in Theorem 5.1 permits the following extension of the class of (completely regular Hausdorff) realcompact spaces.

Definition. A topological space $X$ is realcompact if every stable family of closed subsets with the finite intersection property has nonempty intersection.

The following results extend some of the well-known properties of realcompact completely regular Hausdorff spaces to the general case. In applying the terms "compact" and "regular" to a space, we do not imply that the space is Hausdorff.

THEOREM 5.2. (a) Any compact space is realcompact.

(b) A pseudocompact realcompact space is compact.

(c) Every closed subspace of a realcompact space is realcompact.

(d) Any product of realcompact spaces is realcompact.

Proof. The first three statements are immediate.

For the product theorem, we adapt the proof given in [GJ, 8.12]. Let $X=\prod_{\alpha} X_{\alpha}$, where each space $X_{\alpha}$ is realcompact, and let $\mathscr{F}$ be a maximal stable family of closed subsets of $X$ with the finite intersection property. For each $\alpha$, let $\mathscr{F}_{\alpha}$ be the family of all closed subsets $G$ of $X_{\alpha}$ such that $\pi_{\alpha}{ }^{\leftarrow}[G] \in \mathscr{F}$. If $g \in C\left(X_{\alpha}\right)$, then $g \circ \pi_{\alpha} \in C(X)$; choose $F \in \mathscr{F}$ and a closed bounded subset $H$ of the line such that $F \subseteq\left(g \circ \pi_{\alpha}\right)^{\leftarrow}[H]$. Then $\pi_{\alpha}{ }^{-}\left[g^{\leftarrow}[H]\right] \in \mathscr{F}$, so $g$ is bounded on $g^{\leftarrow}[H] \in \mathscr{F}_{\alpha}$. Thus $\mathscr{F}_{\alpha}$ is a stable family of closed subsets of $X_{\alpha}$ with the finite intersection property. For each $\alpha$, choose $x_{\alpha} \in \bigcap \mathscr{F}_{\alpha}$, and let $x$ denote the point $\left(x_{\alpha}\right)$ of $X$. Let $F=\bigcup_{i} \pi_{\alpha_{i}}{ }^{-}\left[G_{i}\right]$ be a basic closed set with $F \in \mathscr{F}$, where $G_{i}$ is a closed set in $X_{\alpha_{i}}, 1 \leqq i \leqq n$. By the maximality 
of $\mathscr{F}$, one of the sets $\pi_{\alpha_{i}}{ }^{\leftarrow}\left[G_{i}\right]$ belongs to $\mathscr{F}$; hence $G_{i} \in \mathscr{F}_{\alpha_{i}}$, so $x_{\alpha_{i}} \in G_{i}$ and $x \in F$. Hence $\bigcap \mathscr{F} \neq \varnothing$.

For arbitrary spaces we have the following weakened form of [GJ, 8.16].

THEOREM 5.3. A space which is the union of a compact subspace and a C-embedded realcompact subspace is realcompact.

Proof. Let $X=K \cup Y$ with $K$ compact and $Y$ realcompact and $C$-embedded in $X$. Let $\mathscr{F}$ be a stable family of closed subsets of $X$ with the finite intersection property. We may assume that $\mathscr{F}$ is closed under finite intersections. If every member of $\mathscr{F}$ meets $K$, then clearly $\mathscr{F}$ has nonempty intersection. If some member of $\mathscr{F}$ does not meet $K$, then $\mathscr{G}=\{F \cap Y: F \in \mathscr{F}\}$ is a family of closed subsets of $Y$ with the finite intersection property. Clearly $\mathscr{G}$ is stable; hence $\bigcap \mathscr{G} \neq \varnothing$ and also $\bigcap \mathscr{F} \neq \varnothing$.

The following result extends [GJ, 8A]. Another extension will be given in Corollary 6.2.

THEOREM 5.4. A C-embedded realcompact subspace of a Hausdorff space is closed.

Proof. Let $X$ be a $C$-embedded subspace of a Hausdorff space $T$ that is not closed and let $p$ be a limit point of $X$ not in $X$. Let $\mathscr{F}$ be the family of all (closed) subsets of $X$ of the form $F \cap X$, where $F$ is a closed neighborhood of $p$ in $T$. It follows that $\mathscr{F}$ is a stable family with the finite intersection property, but empty intersection, and hence $X$ is not realcompact.

Let $X$ be any space, and let $X_{\mathrm{cr}}$ be the completely regular Hausdorff space obtained in [GJ, 3.9], with $C\left(X_{\mathrm{cr}}\right) \approx C(X)$.

THEOREM 5.5. If $X$ is realcompact, then $X_{\mathrm{cr}}$ is also realcompact. The converse is not true.

Proof. Let $\mathscr{F}$ be a stable family of closed subsets of $X_{\text {cr }}$ with the finite intersection property. Put $\mathscr{G}=\left\{\tau^{\leftarrow}[F]: F \in \mathscr{F}\right\}$, where $\tau: X \rightarrow X_{\text {cr }}$ is the canonical mapping. Clearly $\mathscr{G}$ is a stable family of closed subsets of $X$ with the finite intersection property; hence $\bigcap \mathscr{G} \neq \varnothing$ and thus $\bigcap \mathscr{F} \neq \varnothing$. Hence $X_{\text {cr }}$ is realcompact.

EXAMPLE 3. For a counterexample to the converse, let $X$ be the regular space of $\left[\mathrm{H}_{4}\right]$ on which every real continuous function is constant. It is shown in $\left[\mathrm{H}_{4}\right]$ that $X$ is $T_{1}$, hence not compact, and thus since it is pseudocompact, it is not realcompact. However, $X_{\text {cr }}$ consists of a single point, and is thus realcompact.

The failure of the converse is not to be lamented. It is desired that Theorem 3.1 be true in the general case, and clearly the space $X$ of this example is not $\psi$-compact, and hence must not be realcompact under any definition we might adopt. Since $C(X) \approx C\left(X_{\mathrm{cr}}\right)$, no definition of realcompactness that preserves Theorem $3.1 \mathrm{can}$ be algebraically invariant.

A different extension of realcompactness has been given in [D], but under which some of the properties of realcompact spaces, for example Theorem 3.1, are lost. 
6. The realcompactification of an arbitrary space. In this section we obtain a generalization of the Hewitt realcompactification $v X$ of a completely regular Hausdorff space (see [GJ, Chapter 8]), except that we are able to prove extendability only for continuous mappings into regular realcompact spaces.

THEOREM 6.1. Every topological space $X$ has a realcompactification $v X$, which is a realcompact space in which $X$ is dense and $C$-embedded. Every continuous mapping from $X$ into any regular realcompact space may be extended continuously over $v X$. The space $X$ itself is realcompact if and only if $X=v X$.

Proof. We shall omit those details which are the same as steps in the proof of the existence of the Stone-Čech compactification of a completely regular Hausdorff space given in [GJ, Chapter 6], on which this proof is patterned.

For any $x \in X$, we shall let $\mathscr{F}^{x}$ denote the family of all closed subsets of $X$ that contain $x$. Clearly $\mathscr{F} x$ is stable. (Note that $X$ is a $T_{0}$-space if and only if the correspondence $x \rightarrow \mathscr{F}^{x}$ is one-one. Also, a $T_{0}$-space is a $T_{1}$-space if and only if each $\mathscr{F} x$ is a maximal family of closed subsets with the finite intersection property.)

Let $v X-X$ be an index set for the collection $\left\{\mathscr{F}^{p}: p \in v X-X\right\}$ of all maximal stable families of closed subsets of $X$ with the finite intersection property and empty intersection.

For any closed set $F \subseteq X$, put $\bar{F}=\{p \in v X: F \in \mathscr{F} p\}$; topologize $v X$ by taking these sets $\bar{F}$ as a base for the closed sets. We have $\bar{F} \cap X=F$ and $\bar{F}=\mathrm{cl}_{v X} F$; thus $X$ is a subspace of $v X$ and is dense in $v X$.

Let $\tau: X \rightarrow Y$ be a continuous mapping, with $Y$ regular and realcompact. For any $p \in v X$, let $\mathscr{G}^{p}$ be the family of all closed subsets $G$ of $Y$ such that $\tau^{\leftarrow}[G] \in \mathscr{F}^{p}$. Let $g \in C(Y)$; then $g \circ \tau \in C(X)$, so $g \circ \tau$ is bounded on some member $F$ of $\mathscr{F}^{p}$. Thus $F \subseteq(g \circ \tau)^{\leftarrow}[H]$, where $H$ is some closed bounded subset of the line. It follows that $\tau^{\leftarrow}\left[g^{\leftarrow}[H]\right] \in \mathscr{F}^{p}$ and hence $g^{\leftarrow}[H] \in \mathscr{G}^{p}$; thus $g$ is bounded on a member of $\mathscr{G}^{p}$. Hence $\mathscr{G}^{p}$ is a stable family of closed subsets of $Y$ with the finite intersection property. Choose $y \in \bigcap \mathscr{G}^{p}$ and put $\tau^{0} p=y$ (if $p \in X$, then $\tau p \in \bigcap \mathscr{G}^{p}$ and we choose $y=\tau p$; thus $\tau^{0}$ extends $\tau$ ).

To establish the continuity of $\tau^{0}$ at a point $p \in v X$, let $V$ be any closed neighborhood of $\tau^{0} p$ in $Y$. Put $T=Y-$ int $V, A=\tau^{\leftarrow}[V], B=\tau^{\leftarrow}[T]$, and $U=v X-\bar{B}$. Since $\tau^{0} p \notin T$, we have $T \notin \mathscr{G}^{p}$, so $B \notin \mathscr{F}^{p}$ and $U$ is a neighborhood of $p$ in $v X$. We have $V \cup T=Y$, so $\bar{A} \cup \bar{B}=v X$ and $U \subseteq \bar{A}$. Now if $q \in U$, then $q \in \bar{A}$ and $A \in \mathscr{F}^{q}$; hence $V \in \mathscr{G}^{q}$ and $\tau^{0} q \in V$. Hence $\tau^{0}[U] \subseteq V$, and $\tau^{0}$ is continuous at $p$.

To show that $v X$ is realcompact, consider any stable family $\mathscr{G}$ of closed subsets of $v X$ with the finite intersection property. Let $\mathscr{F}$ be the family of all closed subsets $F$ of $X$ such that $\bar{F}$ contains some member of $\mathscr{G}$. Let $f \in C(X)$ and choose $G \in \mathscr{G}$ such that $f^{v}$ is bounded on $G$. Say $\left|f^{v}\right|<n$ on $G$. Put $A=\{x \in \mathbf{R}:|x| \leqq n\}$, $B=\{x \in \mathbf{R}:|x| \geqq n\}$, and $F=f^{\leftarrow}[A]$. Let $p \in G$ and suppose $F \notin \mathscr{F}^{p}$. Since $A \cup B=\mathbf{R}$, we have $F \cup f^{-}[B]=X$, and thus $f^{-}[B] \in \mathscr{F}^{p}$. It follows from the definition of the extension $f^{v}$ that $f^{v}(p) \in B$, contradicting the choice of $p$ and $n$. Hence $F \in \mathscr{F}^{p}$, so 
$p \in \bar{F}$. Thus $G \subseteq \bar{F}$, and $F \in \mathscr{F}$. Since $f$ is bounded on $F$, it follows that $\mathscr{F}$ is a stable family of closed subsets of $X$ with the finite intersection property; hence $\mathscr{F} \subseteq \mathscr{F}^{p}$ for some $p \in v X$ and we have $p \in \bar{F}$ for every $F \in \mathscr{F}$. Since the sets $\bar{F}$ form a base for the closed subsets of $v X$, we have $p \in \bigcap \mathscr{G}$. Hence $v X$ is realcompact.

The following result extends the characterization given in [GJ, $8 \mathrm{~A}$ ].

COROLlary 6.2. A regular Hausdorff space is realcompact if and only if it is dense and $C$-embedded in no other Hausdorff space.

Proof. The necessity is a special case of Theorem 5.4. Conversely, if $X$ is regular and Hausdorff but not realcompact, then for any $p \in v X-X$, the space $X \cup\{p\}$ is Hausdorff.

EXAMPLE 4. There is no possibility of finding a Hausdorff realcompactification for an arbitrary Hausdorff space $X$, even when $X$ is regular. For, let $X$ be Hewitt's space discussed in Example 3. If $X$ is dense in some Hausdorff realcompact space $T$, then every real continuous function on $T$ is also constant; hence $T$ is pseudocompact, hence compact, and the subspace $X$ is completely regular, which is absurd.

\section{REFERENCES}

$\left[\mathrm{C}_{1}\right] \mathrm{W}$. W. Comfort, On the Hewitt realcompactification of a product space, Trans. Amer. Math. Soc. 131 (1968), 107-118. MR 36 \#5896.

$\left[\mathrm{C}_{2}\right]-$, A theorem of Stone-Cech type, and a theorem of Tychonoff type, without the axiom of choice; and their realcompact analogues, Fund. Math. 63 (1968), 97-110. MR 38 \#5174.

[D] R. F. Dickman, Jr., Compactifications and realcompactifications of arbitrary topological spaces (to appear).

[F] Z. Frolik, Applications of complete families of continuous functions to the theory of $Q$ spaces, Czechoslovak Math. J. 11 (86) (1961), 115-133. MR 23 \#A4122.

[GJ] L. Gillman and M. Jerison, Rings of continuous functions, University Series in Higher Math., Van Nostrand, Princeton, N. J., 1960. MR 22 \#6994.

$\left[\mathrm{H}_{1}\right] \mathrm{A} . \mathrm{W}$. Hager, On the tensor product of function rings, Doctoral Dissertation, Pennsylvania State University, University Park, Pa., 1965.

[ $\left.\mathrm{H}_{2}\right]$ D. Harris, An internal characterization of real-compactness, Canad. J. Math. (to appear).

$\left[\mathrm{H}_{3}\right]$ A. Hayes, Alexander's theorem for realcompactness, Proc. Cambridge Philos. Soc. 64 (1968), 41-43. MR 36 \#4524.

[H $\left.{ }_{4}\right]$ E. Hewitt, On two problems of Urysohn, Ann. of Math. (2) 47 (1946), 503-509. MR 8, 165.

$\left[\mathrm{H}_{5}\right]-$, Rings of real-valued continuous functions. I, Trans. Amer. Math. Soc. 64 (1948), 45-99. MR 10, 126.

[K ${ }_{1}$ ] I. Kaplansky, Topological rings, Amer. J. Math. 69 (1947), 153-183. MR 8, 434.

$\left[\mathrm{K}_{2}\right]$ C. W. Kohls, Ideals in rings of continuous functions, Fund. Math. 45 (1957), 28-50. MR 21 \#1517.

[M] M. Mandelker, Round z-filters and round subsets of $\beta X$, Israel J. Math. 7 (1969), 1-8. MR 39 \#6264.

$\left[\mathrm{N}_{1}\right]$ S. Negrepontis, Baire sets in topological spaces, Arch. Math. (Basel) 18 (1967), 603-608. MR 36 \#3314.

$\left[\mathrm{N}_{2}\right]$ N. Noble, Ascoli theorems and the exponential map, Trans. Amer. Math. Soc. 143 (1969), 393-412. MR 40 \#1978. 
[P] D. Plank, On a class of subalgebras of $C(X)$ with applications to $\beta X-X$, Fund. Math. 64 (1969), 41-54. MR 39 \#6266.

$\left[R_{1}\right] \mathrm{S}$. M. Robinson, The intersection of the free maximal ideals in a complete space, Proc. Amer. Math. Soc. 17 (1966), 468-469. MR 32 \#6401.

$\left[\mathrm{R}_{2}\right]-$, A note on the intersection of free maximal ideals, J. Austral. Math. Soc. 10 (1969), 204-206. MR 39 \#7438.

[W] M. D. Weir, A net characterization of realcompactness (to appear).

New Mexico State University,

Las Cruces, New Mexico 88001 